This document is the accepted manuscript version of the following article:

Seppälä, 0., \& Jokela, J. (2016). Do coinfections maintain genetic variation in parasites? Trends in Parasitology, 32(12), 930-938. https://doi.org/10.1016/j.pt.2016.08.010

This manuscript version is made available under the CC-BY-NC-ND 4.0 license http:// creativecommons.org/1icenses/by-nc-nd/4.0/

\title{
$1 \quad$ Do coinfections maintain genetic variation in parasites?
}

2

3 Otto Seppälä, ${ }^{1,2 *}$ and Jukka Jokela ${ }^{1,2}$

$4 \quad{ }^{1}$ Eawag, Swiss Federal Institute of Aquatic Science and Technology, 8600 Dübendorf,

$5 \quad$ Switzerland

$6 \quad{ }^{2}$ ETH Zürich, Institute of Integrative Biology (IBZ), 8092 Zürich, Switzerland

$7 \quad *$ Correspondence: otto.seppaelae@eawag.ch (O. Seppälä)

8

9

10 
Host individuals are often infected with multiple, potentially interacting parasite species and genotypes. Such coinfections have consequences for epidemiology, disease severity and evolution of parasite virulence. As fitness effects of coinfection can be specific to interacting parasite genotypes, coinfections may induce high fitness variation among parasite genotypes. We argue that such interactions can be an important mechanism maintaining genetic variation in parasite traits such as infectivity and virulence. We also argue that such interactions may slow coevolutionary dynamics between hosts and parasites. This is because instead of depending only on host genotype, parasite fitness may be determined by average infection success across all coinfection scenarios.

\section{Maintenance of genetic variation in host-parasite interactions}

High within-population genetic variation in host resistance (see Glossary) and immune function [1-6], as well as in parasite infectivity [2, 4, 6-8], is commonly found across a broad range of host-parasite interactions. This seems paradoxical because such traits are important components of fitness, and heritable genetic variation in them should be eroded by natural selection [9]. From host perspective, strong selection on resistance to infections can be expected as parasites reduce host survival and fecundity [10]. Similarly, successful infection and within-host growth is required for positive fitness in parasites. Thus, selection should sweep the best gene variants to fixation in the host and parasite populations, and lead to low genetic variation in defense and infection related traits. This, however, is not what is observed [1-8]. The question is what maintains genetic variation in host and parasite traits?

Negative frequency-dependent selection has been discussed as one key factor maintaining genetic variation in host and parasite populations [11]. In coevolutionary models, parasites that are able to infect the most common host genotype(s) have the highest fitness, rendering a fitness advantage to rare host genotypes $[12,13]$. This leads to fluctuating host 
and parasite genotype frequencies known as the Red Queen dynamics (RQD, Box 1), which can maintain genetic variation in the interacting populations [13]. Being very difficult to study, RQD has only rarely been demonstrated in natural populations $[14,15]$. However, a tight genetic specificity determining the outcome of infections, and thus opportunity for RQD, has been shown in many systems $[2,4,16]$, suggesting that RQD might be more common than directly shown.

Many host and parasite traits are, however, not specific to the genetic identity of the interacting partners (e.g. nonspecific immune function) $[17,18]$. Such traits evolve through selection on heritable additive genetic variance $[3,5]$ and call for other mechanisms than negative frequency-dependent selection to explain the maintenance of genetic polymorphism. For example, spatial and temporal heterogeneity of the environment shaping the outcome of host-parasite interactions is widely acknowledged to promote maintenance of genetic variation in host defenses against parasites $[11,19]$. This is because genetic variation in host resistance often interacts with environmental variation so that genotype rank order for susceptibility to infections or immune function changes in relation to environmental conditions [i.e. different genotypes are favored by parasite-mediated selection in different environments; genotype-by-environment $(\mathrm{G} \times \mathrm{E})$ interaction; Figure 1] [20-23]. Studies investigating $\mathrm{G} \times \mathrm{E}$ interactions in determining host resistance have covered a range of environmental factors and discovered strong effects of primary environmental parameters [11, 19]. For example, in a study by Mitchell and coworkers [21], clone rank order of the aquatic crustacean Daphnia magna for infection by the bacterial parasite Pasteuria ramosa, as well as for fitness losses caused by the infection, changed dramatically depending on water temperature. Such strong $\mathrm{G} \times \mathrm{E}$ interactions indicate context dependent fitness variation among genotypes that can effectively maintain genetic polymorphism. How significant $\mathrm{G} \times \mathrm{E}$ interactions are in maintaining genetic variation in parasites is still largely unknown. So far, 
genotype-specific responses of parasites to ambient temperature as well as to food quality for hosts in determining parasite performance have been reported in few systems [6, 8, 24-26]. In this opinion article, we review the recent research on genetic specificity in interactions among coinfecting parasites, and argue that fitness variation created by such interactions in variable coinfecting communities in nature may be one key mechanism maintaining genetic variation in parasite populations. Furthermore, we propose that genetically heterogeneous interactions with direct consequences on parasite fitness may influence also other evolutionary processes such as evolution of virulence and host-parasite coevolutionary dynamics. Research on evolutionary consequences of coinfections for parasite fitness and disease dynamics is already an important field in host-parasite research and we believe that this research direction has potential to address some of the key open questions in host-parasite evolutionary ecology.

\section{Coinfections as a source of fitness variation in parasites}

In nature, individual hosts are often simultaneously infected with multiple parasite species and genotypes that interact $[27,28]$. Since hosts represent the primary environment for parasites, the presence of coinfecting parasite species/genotypes can potentially modify their fitness the same way as the above-mentioned environmental factors alter the performance of host genotypes. In fact, coinfections have recently been shown to induce high variation in the relative fitness of parasite genotypes through genetically specific interactions among coinfecting partners [29-32].

For example, Diplostomum (Trematoda) parasites infecting freshwater fishes are typically observed in multiple-species [33] and in multiple-genotype [34] infections. In this system, interactions between genotypes of two species, Diplostomum pseudospathaceum and Diplostomum gasterostei, have a strong genetic basis that modifies the relative infection 
success of D. pseudospathaceum (but not of D. gasterostei) genotypes between single and multiple infections [30, 31]. This alters the rank order of D. pseudospathaceum genotypes for infectivity depending on the presence/absence of $D$. gasterostei in the same fish individuals. Thus, the effect of coinfections on the relative fitness of parasites and maintenance of genetic polymorphism can have similar effects to the $G \times E$ interactions affecting host resistance. The effect of coinfections on the infection success of D. pseudospathaceum is, however, dose dependent, and observed in experimental exposures only when a high exposure dose (100 infective stages of each parasite species per fish) is used [31]. With a lower exposure dose (50 infective stages of each parasite species per fish), the effect of coinfections appears even more complex. In low exposure dose, the effect of coinfection on parasite performance becomes dependent on the genetic identity of both interacting partners, and the relative fitness of $D$. pseudospathaceum genotypes varies also among different multiply infected host individuals [31]. Since fish are most likely exposed to low numbers of parasite larvae simultaneously, the latter scenario probably dominates in this system in the wild. Furthermore, the effect of coinfections in changing the fitness rank of Diplostomum parasites is not limited to interactions between closely related taxa. In a recent study, Louhi and coworkers [32] showed that interactions with Flavobacteria strains also modify the infection success of Diplostomum genotypes in a strain-specific manner. These results suggest that although the infectivity of Diplostomum parasites seems genotype specific, there is ample variation in the relative success of different genotypes that depends on coinfections.

In addition to Diplostomum parasites of fish, the competitive ability of strains of the protozoan Eimeria tenella in double-genotype infections in chickens has been shown to be difficult to predict from their performance in single-genotype infections [29]. To our knowledge, the only test on the potential of coinfections to maintain genetic diversity in natural parasite populations comes from a tick-transmitted bacterium Borrelia afzelii infecting 
bank voles. In this system, Andersson and coworkers [35] demonstrated that coinfections with multiple bacterium strains are more common than expected by chance, and most importantly, that strains that are antigenically more different are also more strongly associated [35]. Such an effect may maintain genetic polymorphism and take place if increased heterogeneity of infection leads to less effective host defenses $[36,37]$. Taken together, the above examples suggest that genetically specific interactions in coinfections can create high fitness variation in parasites. This can be potentially very important in maintaining genetic polymorphism in natural parasite populations that experience high between-host variation in the genetic composition of coinfecting parasite communities (i.e. different host individuals are infected with different parasite species and genotypes). This is because such effects can create fitness variation among parasites in a very small scale (among host individuals) compared to other environmental factors (e.g. ambient temperature) that have similar effects on parasites across numerous hosts in a shared locality.

Experimental studies providing most of the evidence for fitness variation generated by coinfections are, however, limited only to interactions between two coinfecting partners [29-32]. Although the described effects are strong, we propose that the role of coinfections in inducing fitness variation in natural parasite populations may be even larger. This is because within-host parasite communities of many host species consist of a much higher number of different parasite species and genotypes than what have been investigated experimentally [27, $34,35,38]$. Furthermore, these communities typically show high variation among host individuals. Thus, interactions among coinfecting parasites are potentially very complex and variable in natural settings. Since fitness variation induced by them can be assumed to increase with the number of interacting partners (Box 2), coinfections may even become one of the most significant factors maintaining genetic polymorphism in parasites. Unfortunately, it is still largely unclear how common such heterogeneous interactions are in nature. This 
would be important to understand both across various study systems and parasite traits to reveal the full potential of coinfection in maintaining genetic variation in parasite populations.

\section{Implications for evolution of virulence}

To our knowledge, studies on the genetic specificity in interactions among coinfecting parasites have largely focused on parasite infectivity (see above). Heterogeneous interactions could, however, also affect numerous other parasite traits. One of such traits that has also received wide interest in evolutionary ecological research is parasite virulence $[32,39]$. In general, interactions among coinfecting parasites have been proposed to promote evolution of parasite traits that relate to high host exploitation, thus leading to high virulence [40, 41]. In single infections, evolution of host exploitation by parasites is predicted to arise from its positive and negative effects on different components of parasite fitness. Parasites need to exploit host resources for their within-host growth and reproduction in order to produce enough transmission stages to enable between-host transmission. High host exploitation rate, however, increases the mortality of their present hosts, thus reducing transmission opportunities (trade-off hypotheses) [42, 43]. Therefore, parasite fitness is often assumed to be highest at intermediate levels of virulence. In coinfections, however, parasites can compete for the same within-host resources. Several theoretical models predict parasites with the highest host exploitation rate to be the most successful in exploitation competition, which then leads to evolution towards higher optimal level of virulence when coinfections are common [44-46]. Despite of strong theoretical support, empirical evidence for coinfections selecting for increased virulence is, however, scarce [47-49]. In addition to exploitation competition, coinfecting parasites can interact through several other mechanisms such as interference and host immune responses [50-52]. When competition among parasites takes place through interference mechanisms rather than 
exploitation, the predictions of the effect of coinfections on the evolution of virulence can change dramatically $[40,46,51,53]$. This is because interference competition requires parasite traits that harm competitors such as production of toxins. Because within-host selection under interference competition can favor high investment in interference-related traits, and such traits are likely to be energetically costly to the producer (e.g. reduce growth and/or reproduction), parasite virulence may evolve towards lower optimal level in such systems. Therefore, it is highly important to understand under which kind of competitive interactions parasites occur in the wild, and if this varies across different host individuals depending on the coinfecting parasite community. For example, if parasites compete for common host resources exploitatively in multiple genotype infections, but by interference in multiple species infections, this can lead to considerable variation in which parasite traits are under selection in different host individuals, and thus modify selection on virulence $[40,53]$. Such context dependence could favor different host exploitation strategies in single infections, in multiple-genotype infections, and in multiple-species infections. This variation could maintain genetic polymorphism in virulence in a parasite population and slow down its evolution towards the direction of the most dominant selection gradient.

The best evidence supporting this idea comes from mixed infections of two nematode species (Steinernema spp.) infecting insects [49]. These nematodes live in soil and have bacterial symbionts that enable parasitism. Nematodes release bacteria into their hosts, which increases virulence and enhances nematode reproduction after host death [54]. Interestingly, the interaction between the nematode and the bacteria is specific, nematode performance being reduced with nonnative bacteria [55]. These symbionts also produce toxins that kill other bacteria, thus enabling interference competition between coinfecting nematodes. The ability of bacterium strains to interfere with each other, however, varies greatly. This defines the competitive ability of nematodes and also selection on virulence in 
multiple infections. When nematodes carry bacteria that do not release toxins, the most virulent nematode is the most successful [49]. However, nematodes carrying bacteria able to inhibit the bacteria of their competitor have a competitive advantage independently of their virulence [49]. Similar effects are proposed also in the fungus Metarhizium anisopliae that infects insects [48]. Under intraspecific competition, strains with the highest virulence show the highest competitive ability (i.e. the smallest reduction in performance when compared to single infections) [48]. In mixed-species infections with a nematode, Steinernema feltiae, less virulent strains of the fungus show least reduction in their performance [48]. This study, however, does not directly test the relationship between parasite virulence and fitness. Although the most virulent strains show the largest proportional reduction in their performance in mixed-species infections, their fitness may still remain higher than that of the less virulent strains that show smaller proportional reduction in performance.

\section{Implications for host-parasite coevolution}

In addition to the effects of genetically specific interactions among coinfecting parasites on evolution of specific parasite traits, such interactions can potentially have wider coevolutionary consequences on host-parasite interactions. The RQD theory describes parasite fitness as a specific match/mismatch among parasite and host genes that allow/prevent infection $[12,13]$ (Box 1). Here, the fitness of a parasite genotype is determined by the frequency of the matching host genotype, and the evolutionary dynamics are governed by frequency-dependent selection in the coupled host and parasite populations. Evolutionary dynamics determine the probability for the same gene combinations of the parasite and the host to meet in the next generation. Contrary to the RQD theory, quantitative genetic theory of phenotypic evolution that relies on additive genetic variance considers both 
genetic and non-genetic variation [56]. However, quantitative genetics is applied rarely in studies on host-parasite coevolution $[10,57]$.

In the case of coinfections, empirical data that we reviewed above suggest that the fitness of each parasite genotype is affected by the genotypes of coinfecting partners. This is a recognized form of among-individual epistatic fitness variation where genes in another individual determine the value of one's own genes [58]. However, the role of this epistatic component in determining the fitness of an individual parasite is largely unpredictable owing to high variation in coinfecting parasite communities among different hosts. This is because in horizontal transmission, coinfecting parasite genotypes are uncoupled in each generation and there are very few ecological and evolutionary processes that would secure them sharing a host in the next generation. Therefore, the effects of coinfection on fitness are unpredictable and selection is unlikely to operate on such variation.

Parasite life-history traits could, however, respond to stochastic fitness variation by favoring high reproductive output that ensures dispersion of produced offspring across multiple host individuals and thus coinfection scenarios. In such bet-hedging strategies [59, 60], the parasite bets against multiple coinfection scenarios by sending a high number of offspring to interact with other parasite species/genotypes in numerous host individuals. Selection favors parasite genotypes that have the highest average infection success across various interactions with other players. In other words, high number of transmission stages may be the best way to deal with unpredictability of fitness. Not surprisingly, many parasite life-cycles exhibit this type of reproductive effort [61]. Such strategies do not rely on specific coevolution between host and parasite, but parasite infection success can behave as a quantitative trait in which variation induced by coinfecting parasites can be seen as noise around the mean infection success which is predictable for each parasite genotype. At the 
moment, however, we do not know how such unpredictable interactions among coinfecting parasites influence Red Queen type coevolutionary dynamics.

\section{Concluding remarks}

253 Simultaneous infections with multiple parasite species and genotypes are a rule in nature [27, 28]. Interactions among coinfecting parasites are acknowledged to contribute to many important ecological and evolutionary processes from epidemiological dynamics to evolution of virulence. Recent evidence shows that the outcome of interactions among coinfecting parasites can be highly specific to the genetic identity of the interacting partners $[29-32,35]$. This induces high variation in the relative fitness of parasite genotypes between singly and multiply infected hosts, as well as among different multiply infected host individuals. The latter effect arises from the differences in the coinfecting communities among individual hosts. Moreover, these effects can be assumed to become stronger when the number of interacting parasites in a single host individual increases (Box 2.). Due to all these aspects we argue that interactions among coinfecting parasites are a key mechanism maintaining genetic variation in parasites.

Additionally, we propose that variation in interactions among coinfecting parasites may significantly alter the predictions of two key processes in host-parasite evolution, namely evolution of parasite virulence and coevolutionary dynamics. Classically, coinfecting parasites are assumed to interact through exploitation competition and selection to favor high virulence $[44,45,47]$. However, this prediction changes dramatically when parasites interact, for example, through interference [51]. Therefore, understanding how selection operates on virulence requires understanding the variation in coinfecting communities experienced by the

272 whole parasite population. Specifically, we propose that it would be important to measure

273 how variation in host exploitation/virulence covaries with parasite fitness across hosts 
experiencing different parasite communities. This can be studied experimentally by manipulating the parasite community structure. If covariance becomes weak due to stochasticity introduced by the parasite community structure, it complicates predictions for evolution of virulence. If the covariance remains strong, predictions for evolution of virulence are robust. Furthermore, the RQD theory of host-parasite coevolution predicts parasite fitness to be determined by a match/mismatch of host and parasite genes $[12,13]$ (Box 1).

Unpredictability in parasite fitness caused by coinfections may significantly modify such coevolutionary interactions. Due to commonness of coinfections, high variation in the outcome of interactions among coinfecting parasites, and high reproductive output of most parasite species, we propose that host-parasite coevolution may not rely solely on specific genetic interactions between parasites and their hosts, but selection operates on the average fitness of each parasite genotype across all coinfection scenarios. Thus, parasite infectivity could evolve as a quantitative trait. How genetic interactions among coinfecting parasites influence Red Queen type coevolutionary dynamics between hosts and parasites is, however, not tested. We argue there is a need to incorporate coinfections into future theoretical and empirical studies on host-parasite coevolution.

\section{Acknowledgements}

We thank C. Tellenbach for helpful discussions, and H. Hartikainen, S. Alizon and anonymous reviewers for their comments on the manuscript. The work was supported by the Emil Aaltonen Foundation to O.S., and the Swiss National Science Foundation to O.S. (grants 31003A 140876, 31003A 163318) and J.J. (grant 31003A 129961). 
Additive genetic variance: the magnitude of the phenotypic variance that is due to additive

effects of genes (alleles do not affect the expression of each other). Additive genetic variance determines the degree to which parents' mean phenotype is reflected to offspring.

Coevolution: reciprocal evolution of two or more interacting partners. Evolutionary change in one organism alters the selective pressure on others and vice versa.

Fitness: the relative contribution of an individual to the gene pool of the next generation. In other words, individual's capacity to survive and reproduce when compared to competing individuals.

Infectivity: the ability of parasites to successfully invade and establish into their hosts.

Natural selection: differential survival and reproductive success of individuals due to differences in phenotype (i.e. relationship between phenotypic and fitness variation). Frequency-dependent selection: a type of selection in which the fitness of each phenotype/genotype depends on its frequency relative to other phenotypes/genotypes in the population.

Parasite: an organism that lives in or on another organism (host), and at least partly exploits its resources from the host.

Resistance: the ability of hosts to prevent parasites from infecting them.

Stochastic: either wholly or partially randomly determined.

Virulence: degree of harm (i.e. reduction in fitness) caused to the host by a parasite. 
324 Figure 1. Hypothetical effects of environmental conditions on susceptibility to infection in

325 two host genotypes (each host genotype is connected between the two environments using a

326 reaction norm). (A) Environment affects the susceptibility of hosts to infection, but the effect

327 is similar in both genotypes. (B) Environment affects the susceptibility of hosts to infection,

328 and the effect differs between the genotypes (crossing reaction norms indicate significant

329 genotype-by-environment interaction that changes the fitness rank).

330

331

332

333

334

335

336

337

338

339

340

341

342

343

344

345

346

347

348 


\section{Box 1}

\section{Red Queen hypothesis}

351 Consider interacting host and parasite populations that are both genetically diverse. Also assume that the parasite has to precisely match the host genotype to be able to infect it. Otherwise host immune system will recognize and eliminate the parasite. In such a situation, parasites that can successfully infect the most common host genotypes in the population are most likely to complete their life-cycles and produce propagules to the next generation. Thus, they have the highest fitness and are favored by natural selection. These genotypes will become more frequent in the parasite population over time, thus increasing the risk of infection of the common host types. If these parasites are also virulent (i.e. reduce host fitness), this reduces the reproductive capacity of the common host genotypes. This gives them a selective disadvantage compared to the rare host genotypes that are not under strong selective pressure owing to parasitism. Over time, this difference in selection will drive the frequencies of common host genotypes down, and some previously rare host genotype(s) become common. The change in genotype frequencies in the host population in turn changes the selective pressure on parasites as genotypes that are able to infect the new common host types become favored by selection. The described coevolutionary interaction can lead to cycling of both host and parasite genotype frequencies in the populations (Figure I), thus maintaining genetic variation in both interacting partners. These cycles are, however, not identical, but show a time lag. This is because each partner of the interaction cannot 369 immediately respond to the changes in the genotype frequencies of the other. Instead, a change in host/parasite genotype frequencies will change the selective pressure on 371 parasites/hosts, which can then respond to this selection over time. The term "Red Queen dynamics" for these fluctuations has been adopted by Bell [62] from the Red Queen's 

fast as you can to stay in the same place".

375

Figure I. Hypothetical genotype frequencies of two host (A and B; black and gray solid lines,

377 respectively) and two parasite (a and b; black and gray dashed lines, respectively) genotypes

378 under coevolution. An exact match between the host and parasite genotypes is expected for

379 infection (i.e. parasite a infects host $\mathrm{A}$ and parasite $\mathrm{b}$ infects host $\mathrm{B}$ ).

380

381

382

383

384

385

386

387

388

389

390

391

392

393

394

395

396

397 


\section{Box 2}

\section{Fitness variation in relation to complexity of community}

400

401

402

403

404

405

406

407

408

409

410

411

412

413

414

415

416

417

418

419

420

421

\section{$\underline{\text { Additive effects of interactions }}$}

Consider a parasite genotype that infects its hosts either in single infections or in coinfections with any number of other parasite genotypes (same and/or other species). In single infections, fitness $W$ of this parasite genotype is expected to be equal or larger than zero, and to follow any distribution with positive variance [i.e. $\mathbb{E}(W)>0$ and $\operatorname{Var}(W)>0$ ]. In coinfections, interactions with other parasites additively increase or decrease its fitness and this effect has positive variance [i.e. $\operatorname{Var}(I)>0$ ]. For simplicity, assume that the interactions are not so strongly antagonistic that they would generally reduce the fitness of the focal parasite to zero, which is supported by empirical data [29-32].

Variance in the expected fitness of the focal parasite when coinfecting a host with $k$ other parasites can be written using a rule for variance of the sum of variables (Bienaymé equality [64]), and is

$$
\operatorname{Var}(W+k I)=\operatorname{Var}(W)+k \operatorname{Var}(I)
$$

This variance increases with the number of interacting partners if

$$
\operatorname{Var}(W)+k \operatorname{Var}(I)<\operatorname{Var}(W)+(k+1) \operatorname{Var}(I) .
$$

This simplifies to

$$
0<\operatorname{Var}[I]
$$


which is always true when interactions among coinfecting parasites are variable.

424

425

426

427

\section{$\underline{\text { Multiplicative effects of interactions }}$}

Consider a similar focal parasite as above. Assume that interactions among parasites in coinfections have multiplicative effects, and that these effects follow any distribution with the minimum value of zero (interactions do not lead to negative fitness) and positive variance [i.e. $\operatorname{Var}(I)>0]$

Variance in the expected fitness of the focal parasite when coinfecting a host with $k$ other parasites can be written using a rule for variance of the product of variables [65], and is $\operatorname{Var}\left(W I^{k}\right)=\left[\operatorname{Var}(W)+\mathbb{E}(W)^{2}\right]\left[\operatorname{Var}(I)+\mathbb{E}(I)^{2}\right]^{k}-\mathbb{E}(W)^{2}\left[\mathbb{E}(I)^{2}\right]^{k}$

The equation shows the effect of interactions among parasites on the variance in fitness of the focal parasite including both a 'mixing' term $\operatorname{Var}(I)$ and a 'scaling' term $\mathbb{E}(I)$. More specifically, $\operatorname{Var}(I)$ shows the effect of interactions on variance in parasite fitness due to variation in interactions, whereas $\mathbb{E}(I)$ only rescales parasite performance. $\mathbb{E}(I)$ cannot change the relative fitness of parasite genotypes and it can be removed from the equation by setting it to one. After that, the role of 'mixing' term $\operatorname{Var}(I)$ can be estimated directly and the equation is written as

$\operatorname{Var}\left(W I^{k}\right)=\left[\operatorname{Var}(W)+\mathbb{E}(W)^{2}\right][\operatorname{Var}(I)+1]^{k}-\mathbb{E}(W)^{2}$.

This variance increases with the number of interacting partners if 
$447 \quad\left[\operatorname{Var}(W)+\mathbb{E}(W)^{2}\right][\operatorname{Var}(I)+1]^{k}-\mathbb{E}(W)^{2}<\left[\operatorname{Var}(W)+\mathbb{E}(W)^{2}\right][\operatorname{Var}(I)+1]^{k+1}-$

$448 \quad \mathbb{E}(W)^{2}$.

449

450 The equation can be written as

451

$452\left[\operatorname{Var}(W)+\mathbb{E}(W)^{2}\right][\operatorname{Var}(I)+1]^{k}-\mathbb{E}(W)^{2}<\left[\operatorname{Var}(W)+\mathbb{E}(W)^{2}\right][\operatorname{Var}(I)+1]^{k}[\operatorname{Var}(I)+$

$4531]-\mathbb{E}(W)^{2}$,

454

455 which simplifies to

456

457

$0<\operatorname{Var}(I)$.

458

459 This is always true when interactions among coinfecting parasites are variable.

460

461

462

463

464

465

466

467

468

469

470

471 
4731 Grosholz, E.D. (1994) The effects of host genotype and spatial distribution on trematode parasitism

474 in a bivalve population. Evolution 48, 1514-1524

4752 Carius, H.J., et al. (2001) Genetic variation in a host-parasite association: potential for coevolution

476 and frequency-dependent selection. Evolution 55, 1136-1145

4773 Cotter, S.C., et al. (2004) Costs of resistance: genetic correlations and potential trade-offs in an

478 insect immune system. J Evol Biol 17, 421-429

479

480

481

482

483

484

485

486 specific interactions in a plant-pathogen system. Evolution 59, 2518-2524 5 Schwarzenbach, G.A., et al. (2005) Sex and immunity in the yellow dung fly Scathophaga stercoraria. J Evol Biol 18, 455-463

6 Laine, A.L. (2007) Pathogen fitness components and genotypes differ in their sensitivity to nutrient and temperature variation in a wild plant-pathogen association. J Evol Biol 20, 2371-2378

7 Lively, C.M. (1989) Adaptation by a parasitic trematode to local populations of its snail host. Evolution 43, 1663-1671

8 Vale, P.F. and Little, T.J. (2009) Measuring parasite fitness under genetic and thermal variation. Heredity 103, 102-109

9 Roff, D.A. (1997) Evolutionary quantitative genetics. Chapman \& Hall etc.

10 Seppälä, O. (2015) Natural selection on quantitative immune defence traits: a comparison between theory and data. J Evol Biol 28, 1-9

11 Lazzaro, B.P. and Little, T.J. (2009) Immunity in a variable world. Philos T R Soc B 364, 15-26

12 Haldane, J.B.S. (1949) Disease and evolution. La Ricerca Scientifica (Suppl) 19, 68-76

13 Hamilton, W.D. (1980) Sex versus non-sex versus parasite. Oikos 35, 282-290

14 Decaestecker, E., et al. (2007) Host-parasite 'Red Queen' dynamics archived in pond sediment. Nature 450, 870-U816

15 Jokela, J., et al. (2009) The maintenance of sex, clonal dynamics, and host-parasite coevolution in a mixed population of sexual and asexual snails. Am Nat 174, S43-S53

16 Burdon, J.J. and Jarosz, A.M. (1991) Host-pathogen interactions in natural populations of Linum marginale and Melampsora lini. I. Patterns of resistance and racial variation in a large host population. Evolution 45, 205-217

17 Frank, S.A. (2000) Specific and non-specific defense against parasitic attack. J Theor Biol 202, 283304

18 Moret, Y. (2003) Explaining variable costs of the immune response: selection for specific versus non-specific immunity and facultative life history change. Oikos 102, 213-216

19 Wolinska, J. and King, K.C. (2009) Environment can alter selection in host-parasite interactions. Trends Parasitol 25, 236-244

20 Blanford, S., et al. (2003) Temperature checks the Red Queen? Resistance and virulence in a fluctuating environment. Ecol Lett 6, 2-5

21 Mitchell, S.E., et al. (2005) Host-parasite and genotype-by-environment interactions: temperature modifies potential for selection by a sterilizing pathogen. Evolution $59,70-80$ 22 Lazzaro, B.P., et al. (2008) Genotype-by-environment interactions and adaptation to local temperature affect immunity and fecundity in Drosophila melanogaster. Plos Pathog 4 23 Seppälä, O. and Jokela, J. (2010) Maintenance of genetic variation in immune defense of a freshwater snail: role of environmental heterogeneity. Evolution 64, 2397-2407 24 Fels, D. and Kaltz, O. (2006) Temperature-dependent transmission and latency of Holospora undulata, a micronucleus-specific parasite of the ciliate Paramecium caudatum. Proc R Soc B 273, 1031-1038

25 Bryner, S.F. and Rigling, D. (2011) Temperature-dependent genotype-by-genotype interaction between a pathogenic fungus and its hyperparasitic virus. Am Nat 177, 65-74

26 Sadd, B.M. (2011) Food-environment mediates the outcome of specific interactions between a bumblebee and its trypanosome parasite. Evolution 65, 2995-3001 
27 Holmes, J.C. and Price, P.W. (1986) Communities of parasites. In Community ecology: pattern and process (Kikkawa, J. and Anderson, D.J., eds), pp. 187-213, Blackwell 28 Read, A.F. and Taylor, L.H. (2001) The ecology of genetically diverse infections. Science 292, 10991102

29 Nakamura, T., et al. (1992) Estimation of relative fecundity in Eimeria tenella strains by a mixed infection method. Parasitology 104, 11-17

30 Seppälä, O., et al. (2009) Interactions among co-infecting parasite species: a mechanism maintaining genetic variation in parasites? Proc $R$ Soc $B$ 276, 691-697

31 Seppälä, O., et al. (2012) Reciprocal interaction matrix reveals complex genetic and dosedependent specificity among coinfecting parasites. Am Nat 180, 306-315

32 Louhi, K.R., et al. (2015) Interactions among bacterial strains and fluke genotypes shape virulence of co-infection. Proc R Soc B 282, 20152097

33 Rellstab, C., et al. (2011) Analysis of trematode parasite communities in fish eye lenses by pyrosequencing of naturally pooled DNA. Infect Genet Evol 11, 1276-1286

34 Rauch, G., et al. (2005) How a complex life cycle can improve a parasite's sex life. J Evol Biol 18, 1069-1075

35 Andersson, M., et al. (2013) Multiple-strain infections of Borrelia afzelii: a role for within-host interactions in the maintenance of antigenic diversity? Am Nat 181, 545-554

36 Taylor, L.H., et al. (1998) Virulence of mixed-clone and single-clone infections of the rodent malaria Plasmodium chabaudi. Evolution 52, 583-591

37 Jokela, J., et al. (2000) Dr. Pangloss restrained by the Red Queen - steps towards a unified defence theory. Oikos 89, 267-274

38 Valtonen, E.T., et al. (2001) The structure of parasite component communities in brackish water fishes of the northeastern Baltic Sea. Parasitology 122, 471-481

39 Bose, J. and Schulte, R.D. (2014) Testing G×G interactions between coinfecting microbial parasite genotypes within hosts. Frontiers in Genetics 5, article 124

40 Alizon, S., et al. (2013) Multiple infections and the evolution of virulence. Ecol Lett 16, 556-567

41 Cressler, C.E., et al. (2016) The adaptive evolution of virulence: a review of theoretical predictions and empirical tests. Parasitology 143, 915-930

42 Anderson, R.M. and May, R.M. (1982) Coevolution of hosts and parasites. Parasitology 85, 411426

43 Ewald, P.W. (1983) Host-parasite relations, vectors, and the evolution of disease severity. Annu Rev Ecol Syst 14, 465-485

44 Levin, S. and Pimentel, D. (1981) Selection of intermediate rates of increase in parasite-host systems. Am Nat 117, 308-315

45 Nowak, M.A. and May, R.M. (1994) Superinfection and the evolution of parasite virulence. Proc $R$ Soc $B$ 255, 81-89

46 Choisy, M. and de Roode, J.C. (2010) Mixed Infections and the Evolution of Virulence: Effects of Resource Competition, Parasite Plasticity, and Impaired Host Immunity. Am Nat 175, E105-E118 47 de Roode, J.C., et al. (2005) Virulence and competitive ability in genetically diverse malaria infections. P Natl Acad Sci USA 102, 7624-7628

48 Staves, P.A. and Knell, R.J. (2010) Virulence and competitiveness: testing the relationship during inter- and intraspecific mixed infections. Evolution 64, 2643-2652

49 Bashey, F., et al. (2012) Alternative paths to success in a parasite community: within-host competition can favor higher virulence or direct interference. Evolution 67, 900-907 50 Rellstab, C., et al. (2013) Genotype-specific vs. cross-reactive host immunity against a macroparasite. Plos One 8, e78427 51 Massey, R.C., et al. (2004) Interference competition and parasite virulence. Proc $R$ Soc B 271, 785788 52 Mideo, N. (2009) Parasite adaptations to within-host competition. Trends Parasitol 25, 261-268 53 Bashey, F. (2015) Within-host competitive interactions as a mechanism for the maintenance of parasite diversity. Philos T R Soc B 370 
57554 Sicard, M., et al. (2003) Effect of native Xenorhabdus on the fitness of their Steinernema hosts:

576 contrasting types of interaction. Parasitol Res 91, 520-524

57755 Chapuis, É., et al. (2009) Manifold aspects of specificity in a nematode-bacterium mutualism. J

578 Evol Biol 22, 2104-2117

57956 Lynch, M. and Walsh, B. (1998) Genetics and analysis of quantitative traits. Sinauer

58057 Sorci, G., et al. (1997) Genetics of host-parasite interactions. Trends Ecol Evol 12, 196-200

58158 Wolf, J.B. (2000) Indirect genetic effects and gene interactions. In Epistasis and the evolutionary

582 process (Wolf, J.B., et al., eds), pp. 158-176, Oxford University Press

58359 Frank, S.A. and Slatkin, M. (1990) Evolution in a variable environment. Am Nat 136, 244-260

58460 Beaumont, H.J.E., et al. (2009) Experimental evolution of bet hedging. Nature 462, 90-U97

58561 Schmid-Hempel, P. (2011) Evolutionary parasitology: the integrated study of infections,

586 immunology, ecology, and genetics. Oxford University Press

587

62 Bell, G. (1982) The masterpiece of nature: the evolution and genetics of sexuality. University of

588 California Press

58963 Carroll, L. (1872) Through the looking glass and what Alice found there. Macmillan

59064 Loève, M. (1977) Probability theory I. Springer

59165 Goodman, L.A. (1962) The variance of the product of K random variables. J Am Stat Assoc 57, 54-

59260

593

594 
Seppälä \& Jokela Fig. 1
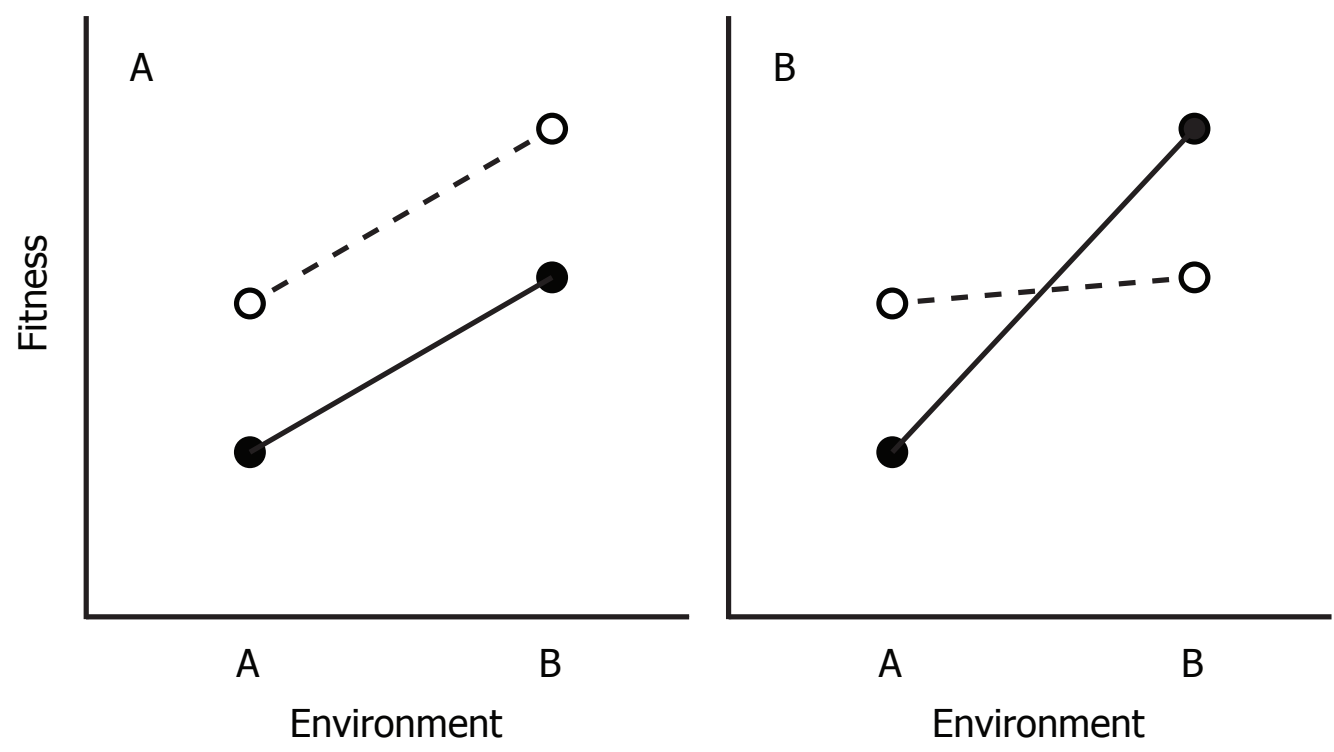
Seppälä \& Jokela Fig. I (Box 1)

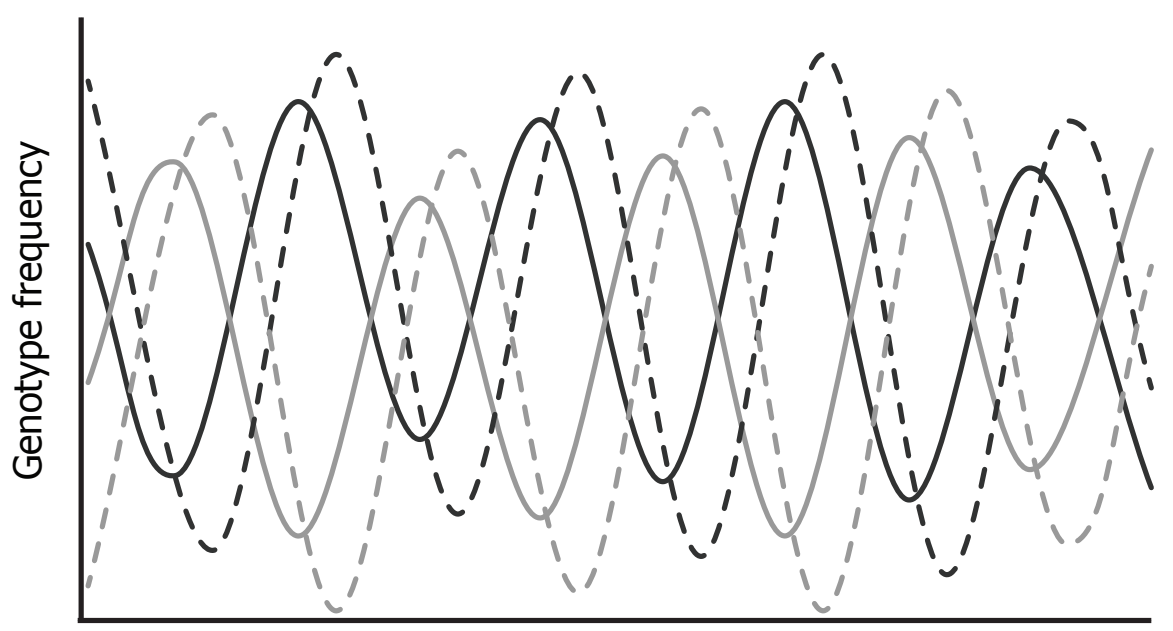

Time 